\title{
SPEED-UP BY THEORIES WITH INFINITE MODELS
}

\author{
R. STATMAN
}

\begin{abstract}
We prove that if $S$ is a finite set of schemata and $A$ is a sentence undecided by $S$ such that $S \cup\{\neg A\}$ has an infinite model then $S \cup\{A\}$ is an unbounded speed-up of $S$ for substitution instances of tautologies. As a corollary, we obtain a conjecture of Parikh's.
\end{abstract}

I. Let $P$ be any of the usual (schematic) formulations of predicate logic with equality, relation and function symbols, and individual constants and let $S$ be a finite set of schemata; by ' $\left.S\right|_{p} ^{n} A$ ' we mean that there is a $P$-derivation of $A$ from (substitution instances of members of) $S$ with $<n$ inferences (lines). We shall prove the following:

THEOREM. Suppose that $A$ is a sentence undecided by $S$ and $S \cup\{\neg A\}$ has an infinite model, then there is a number $n$ such that for each number $m$ there is a substitution instance of a tautology $B$ with $\left.S \cup\{A\}\right|_{p} ^{n} B$ and $\left.S\right|_{p} ^{m} B$.

In short $S \cup\{A\}$ is an unbounded speed-up of $S$ for substitution instances of tautologies.

II. Since for any such $P_{1}$ and $P_{2}$ it is easy to find a function $f$ satisfying $S \mid \frac{k}{P_{1}} B \Rightarrow S \frac{\mid f(k)}{P_{2}} B$, it suffices to set $P=\mathrm{NE}_{1}$, for $\mathrm{NE}_{1}$ the system of natural (deduction) rules for predicate logic with equality (see for example 3.1.6, p. 249 of [3], or the proof of Lemma 2 below). We consider the usual first-order language on $\rightarrow, \perp$ and $\forall$; for the proof it will be convenient to distinguish relation constants from relation parameters, the latter being the arguments of substitutions.

Let $S$ and $A$ be fixed as above; if $C$ is a propositional formula, built up from propositional variables, $\rightarrow$ and $\perp$, a code $F$ of $C$ is any formula $\neg A \rightarrow B$ where $B$ is obtained from $C$ by a 1-1 substitution of equations $u_{i}=v_{i}$ for propositional variables $p_{i}$ such that all the $u_{i}$ and $v_{i}$ are distinct. Note that if $F$ is a code of $C$ then; $S \vDash F . \Leftrightarrow . C$ is a tautology (this only requires that $S \cup\{\neg A\}$ has a $>2$ element model), and $S \cup\{A\} \mid \frac{3}{\mathrm{NE}_{1}} F$. Consequently, it suffices to prove the following:

There is no number $m$ such that if $\neg A \rightarrow B$ is the code of a tautology then

$$
S \cup\{\neg A\} \mid \frac{m}{\mathrm{NE}_{1}} B .
$$

We shall prove the following bounded speed-up result:

There is a function $f$ such that

$$
S \cup\{\neg A\}\left|\frac{n}{\mathrm{NE}_{1}} B \Rightarrow\right| \frac{f(n)}{\mathrm{NE}_{0}} B
$$

Received by the editors April 23, 1979 and, in revised form, February 8, 1980.

AMS (MOS) subject classifications (1970). Primary 02D99. 
for codes of tautologies $\neg A \rightarrow B$, where $\mathrm{NE}_{0}$ is the quantifier-free fragment of $\mathrm{NE}_{1}$ (see 3.1.6, p. 249 of [3]).

Our result then follows from the routine:

Proposition. There is no number $n$ s.t. for codes of tautologies $\neg A \rightarrow B, \frac{n}{\mathrm{NE}_{0}} B$.

III. A quantifier-free formula is said to be 'simple' if each of its prime subformulae has the form

(a) $u=v$, or

(b) $U u_{1} \cdots u_{n}$ for $U$ a relation parameter.

In particular, a simple formula contains no nonlogical constants. Let $\lg (B)={ }_{\mathrm{df}}$ the number of occurrences of logical operations and prime subformulae in $B$ :

LEMMA 1. There is a function $f$ s.t. for simple $B$ if $B$ is valid then $\frac{f(\lg (B))}{\mathrm{NE}_{0}} \quad B$.

Proof. If $\Gamma$ is a set of simple prime formulae let $\operatorname{pm}(\Gamma)=_{\mathrm{df}}$ the number of individual parameters occurring in equations in $\Gamma$ : so, in particular, $\operatorname{pm}(\Gamma)<\overline{\bar{\Gamma}} \cdot 2$. Observe that by the method of 1.5.2 on p. 237 of [3] if $B$ is a simple prime formula and $\Gamma \vDash B$ then $\Gamma \mid \frac{n}{\mathrm{NE}_{0}} B$ for $n=2^{\mathrm{pm}(\Gamma \cup\{B\})}$. In addition if $\Delta$ is a set of simple prime formulae and $\Gamma \vDash W \Delta$ then for some $B \in \Delta, \Gamma \vDash B$.

Now suppose that $\Gamma$ and $\Delta$ are collections of simple formulae and $A \rightarrow B$ is simple then

$$
\begin{gathered}
\Gamma \cup \neg \Delta \cup\left\{A_{1}\right\} \mid \frac{n}{\mathrm{NE}_{0}} \perp \text { and }\left\{A_{2}\right\} \cup \Gamma \cup \neg \Delta \mid \frac{m}{\mathrm{NE}_{0}} \perp \Rightarrow \\
\left\{A_{1} \rightarrow A_{2}\right\} \cup \Gamma \cup \neg \Delta \frac{n+m+4}{\mathrm{NE}_{0}} \perp,
\end{gathered}
$$

and

$$
\left\{A_{1}\right\} \cup \Gamma \cup \neg \Delta \cup\left\{\neg A_{2}\right\}\left|\frac{n}{\mathrm{NE}_{0}} \perp \Rightarrow \Gamma \cup \neg \Delta \cup\left\{\neg\left(A_{1} \rightarrow A_{2}\right)\right\}\right| \frac{n+3}{\mathrm{NE}_{0}} \perp .
$$

Let $\lg (\Gamma)=\Sigma_{A \in \Gamma} \lg (A)$; it follows easily from the above that

$$
\Gamma \vDash W \Delta \Rightarrow \Gamma \cup \neg \Delta \frac{n}{\mathrm{NE}_{0}} \perp
$$

for $n=4^{\lg (\Gamma)+\lg (\Delta)}$ so we can set $f(x)={ }_{\mathrm{df}} 4^{x}+1$.

By a substitution we mean a substitution of relation terms $\lambda x_{1} \cdots x_{n} A$ (with the restriction that each $x_{i}$ occurs in $A$ ) for relation parameters under the definition:

$$
\lambda x_{1} \cdots x_{n} A\left(x_{1} \cdots x_{n}\right) t_{1} \cdots t_{n}=_{\mathrm{df}} A\left(t_{1} \cdots t_{n}\right) \text {. }
$$

For what follows we refer the reader to 4.1-2, pp. 251-255 of [3].

If $\theta$ and $\phi$ are substitutions, then $\theta \phi$ is their composition.

If $F_{i}$ is a finite set of relation terms and $F=F_{1} \cdots F_{n}$, then $\theta \uparrow F$ is the substitution defined by

$$
\begin{aligned}
(\theta \uparrow F) U & =\theta U \text { if } U \text { occurs in a member of some } F_{i}, \\
& =U \text { otherwise. }
\end{aligned}
$$

We say that $\theta$ unifies $F$ if for each $1<i<n, \operatorname{card}\left(\theta^{\prime \prime} F_{i}\right)=1$.

If $F_{i}$ is a finite set of relation terms and $F=F_{1} \cdots F_{n}$, then $\lg (F)$ is the maximum logical complexity of a relation term belonging to some $F_{i}$ and $\operatorname{rel}(F)$ is the total number of relation symbols occurring in members of the $F_{i}$. 
If $\theta$ is a substitution, then $\lg (\theta)={ }_{\mathrm{df}} \max \{\lg (\theta U) ; U \in \operatorname{dom} \theta\}$. Note that $\lg (\theta \phi) \leqslant \lg (\theta) \cdot \lg (\phi)$ and $\lg (\theta F) \leqslant \lg (\theta) \cdot \lg (F)$ where $\theta F={ }_{\mathrm{df}} \theta^{\prime \prime} F_{1} \cdots \theta^{\prime \prime} F_{n}$.

In [3] we proved the following lemma (4.2.1).

Suppose that $F_{i}$ is a finite set of formulae, $F=F_{1} \cdots F_{n}$, and $\theta$ unifies $F$, then there are sustitutions $\phi_{1}, \phi_{2}$ such that

(1) $\phi_{1}$ unifies $F$,

(2) $\theta \uparrow F=\left(\phi_{2} \phi_{1}\right) \uparrow F$, and

(3) $\lg \left(\phi_{1}\right) \leqslant \lg (F)^{m}$ where $m=2^{\operatorname{rel}(F)}$.

Let $S$ be a finite set of schemata.

LEMMA 2. There is a function $f$ s.t. for each $\mathrm{NE}_{1}$-derivation $D$ from $S$ there is an $\mathrm{NE}_{1}$-derivation $D^{*}$ from $S$ and a substitution $\theta$ s.t.

(1) $D=\theta D^{*}$, and

(2) if $A$ occurs in $D^{*}$ then $\lg (A) \leqslant f($ length $(D))$.

Proof. Let $w$ be an injective assignment of 0 -ary relation parameters to the formula occurrences of $D$ and let $S^{*}$ be a finite set of schemata s.t.

(i) each member of $S^{*}$ is a substitution instance in the unrestricted sense of a member of $S$,

(ii) each substitution instance in the unrestricted sense of a member of $S$ is a substitution instance in the restricted sense of a member of $S$. By a copy of a schema we mean the schema up to a permutation of relation parameters. Let $W$ assign to each occurrence of an instance of a member of $S^{*}$ as an assumption in $D$ a copy of the associated member of $S^{*}$ so that different occurrences are assigned copies on disjoint sets of new relation parameters. To each formula occurrence in $D$ we assign a sequence of finite sets of formulae as follows $(B \mapsto X$ means $X$ is assigned to $B$ ).

(a) A formula occurrence which is the conclusion of an inference by a rule other than $=$ is assigned the sets assigned to the inference in 4.2 .2 on p. 253 of [3]. Namely:

(i) If $B$ is the conclusion of

$$
\begin{gathered}
(A) \neq \varnothing \\
\frac{C}{A \rightarrow C}
\end{gathered}
$$

then

$$
B \mapsto\{w(F) \rightarrow w(C): F \in(A)\} \cup\{w(B)\}
$$

(ii) If $B$ is the conclusion of

$$
\frac{C}{A \rightarrow C}
$$

then

$$
B \mapsto\{U \rightarrow w(C), w(B)\}
$$

for $U$ a new 0 -ary relation parameter. 
(iii) If $B$ is the conclusion of

$$
\begin{gathered}
(\neg B) \\
\frac{\perp}{B}
\end{gathered}
$$

then

$$
B \mapsto\{w(\perp), \perp\}\{w(C): C \in(\neg B)\} \cup\{w(B) \rightarrow \perp\} .
$$

(iv) If $B$ is the conclusion of

$$
\frac{A \rightarrow B \quad A}{B}
$$

then

$$
B \mapsto\{w(A) \rightarrow w(B), w(A \rightarrow B)\} .
$$

(v) If $B$ is the conclusion of

$$
\frac{A(u)}{\forall x A(x)}
$$

then

$$
B \mapsto\{w(A(u)), U u\}\{w(\forall x A(x)), \forall x U x\}
$$

for $U$ a new 1-ary relation parameter and $u$ a proper parameter.

(vi) If $B$ is the conclusion of

then

$$
\frac{A}{\forall x A}
$$

$$
B \mapsto\{w(A), U\}\{w(\forall x A), \forall x U\}
$$

for $U$ a new 0 -ary relation parameter.

(vii) If $B$ is the conclusion of

$$
\frac{\forall x A(x)}{A(t)}
$$

then

$$
B \mapsto\{w(A(t)), U t\}\{w(\forall x A(x)), \forall x U x\}
$$

for $U$ a new 1-ary relation parameter, and $x$ actually occurring free in $A(x)$.

(viii) If $B$ is the conclusion of

then

$$
\frac{\forall x A}{A}
$$

$$
B \mapsto\{w(A), U\}\{w(\forall x A), \forall x U\}
$$

for $U$ a new 0 -ary relation parameter.

(b) If $B$ is the conclusion of

$$
\frac{A(a) \quad a \ominus b}{A(b)}
$$

then

$$
B \mapsto\{w(A(a)), U a\}\{w(A(b)), U b\}\{w(a \ominus b), a \ominus b\}
$$

for $U$ a new 1-ary relation parameter. 
(c) If $B$ is the conclusion of

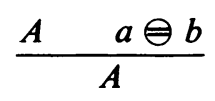

then

$$
B \mapsto\{w(a), U\}\{w(B), U\}\{w(a \ominus b), a \ominus b\}
$$

for $U$ a new 0 -ary relation parameter.

(d) If $B$ is an axiom occurrence then

$$
B \mapsto\{w(B), \forall x(x=x)\} .
$$

(e) If $B$ is an occurrence of an instance of a member of $S^{*}$ as an assumption then

$$
B \mapsto\{w(B), W(B)\}
$$

(f) Otherwise, $B \mapsto\{w(B)\}$, where $a \ominus b$ means ambiguously $a=b$ and $b=a$.

(Below, in order to apply Lemma 4.2.1 of [3] we shall allow relation constants to be the arguments of substitutions.)

Let $F$ be the sequence of all such sets, then there is a substitution $\theta$ such that $\theta$ unifies $F, \theta R=R$ for each relation constant in $F$, and for each occurrence $A$ in $D$ we have $A=\theta w(A)$. By Lemma 4.2.1 of [3, p. 252] there are $\phi_{1} \phi_{2}$ satisfying the conditions (1), (2), and (3) stated there; let $D^{*}$ result from $D$ by replacing each formula occurrence $A$ by $\phi_{1} w(A)$ (and apply a permutation of relation symbols to replace $\phi_{1} R$ by $R$ ).

We now compute an upper bound for $\lg (A)$ for $A$ occurring in $D^{*}$. Let $m=\max \left\{\lg (B): B \in \Gamma^{*}\right\}$ and $k=\max \left\{\operatorname{rel}(B): B \in S^{*}\right\}$, then $\lg (F)<\max \{m, 3\}$ and $\operatorname{rel}(F) \leqslant(2 \cdot \ln (D)+1) \cdot \max \{k, 2\}$. Now $\lg (A) \leqslant \lg \left(\phi_{1}\right) \cdot \lg (F)$; thus there is a linear $e$ s.t. $\lg (A) \leqslant 2_{2}^{e(\ln (D))}$, where $2_{2}^{x}=2^{\left(2^{x}\right)}$.

Proposition. Suppose $S$ has an infinite model then there is a function $f$ s.t. for simple $B$,

$$
S\left|\frac{n}{\mathrm{NE}_{1}} B \Rightarrow\right| \frac{f(n)}{\mathrm{NE}_{0}} B
$$

Proof. Note that if $\theta A=B$ and $B$ is simple then $A$ is simple. Also, if $A$ is simple and $S \vDash A$ then $A$ is valid. The proposition now follows from the lemmas.

III. One special case of the theorem is that Theorem 4 of [2] holds for any of the usual formulations of first-order arithmetic (the corresponding result for the $\varepsilon$-calculus can be found in [1, Theorem 2, p. 107]). More precisely, analysis is an unbounded speed-up of arithmetic for quantifier-free formulae.

\section{REFERENCES}

1. G. Kreisel and H. Wang, Some applications of formalized consistency proofs, Fund. Math. 42 (1955), 101-110.

2. R. Parikh, Some results on the length of proofs, Trans. Amer. Math. Soc. 177 (1973), 29-36.

3. R. Statman, Proof-search and speed-up in the predicate calculus, Ann. Math. Logic 15 (1978), 225-287.

Department of Mathematics, Rutgers University, New Brunswick, New Jersey 08903 